\title{
Plasma Channel Extension by Femtosecond Laser Filamentation with a Circular Aperture Quartz Plate
}

\author{
Yao Li ${ }^{1}$, Ze-lang Zhang ${ }^{1}$, Hai-ying Song 1,* , Xun Liu ${ }^{2}$, Wei Li ${ }^{2}$ and Shi-bing Liu 1,* \\ 1 Strong-field and Ultrafast Photonics Lab, Key Laboratory of Trans-scale Laser Manufacturing Technology, \\ Ministry of Education, Beijing University of Technology, Beijing 100124, China; \\ 2 Beijing Institute of Space Mechanics and Electricity, China Academy of Space Technology, Beijing 100094, \\ China; \\ * Correspondence: hysong@bjut.edu.cn; sbliu@bjut.edu.cn
}

\begin{abstract}
We propose a new approach of extending the laser filament plasma channel. By adding a circular aperture quartz plate before the focusing lens, the extension of the plasma channel is doubled. The effects of different diameters, thicknesses of the circular aperture quartz plate and different pulse energies on the length of the plasma channel were investigated. The experimental results show that the thickness of the quartz plate and the depth of the hole have little effects on the plasma channel of the filament, and the diameter of the hole in the center of the quartz plate has a significant effect on the length of the optical filament. The moving-focus model is used to explain the extension of the optical filament.
\end{abstract}

Keywords: femtosecond laser, plasma channel, filament elongation, circular aperture quartz plate

\section{Introduction}

When the ultrashort pulse laser passes through a special optical system, the focus point of the laser will arrange in a line along the optical axis, the arranged line is called focus line. And when the intensity of the laser focused line is higher than the ionization threshold of the air molecules or atoms, the atmosphere can be breakdown ionized and form plasma, the light generated from the plasma are called laser filament, also filament plasma or plasma channel. Since the self-channeling intense femtosecond laser pulses was first observed by Braun et al. [1], the optical filamentation has been a hot topic since it holds the promise of potential for many applications, such as $\mathrm{THz}$ radiation sources[2], remote radiation sources and triggering[3], spectral manipulations of the laser pulse[4], and guiding of electrical discharges[5][6], atmospheric remote sensing[7], high harmonic generation [8] and attosecond pulse generation[9][10], etc.

Due to the loss of laser energy during laser filament formation, self-focusing and plasma defocusing can no longer be balanced, and the filament disappears at the equilibrium point, so the length and lifetime of the laser filament is limited. Some methods were present to study the filamentation phenomenon in air in experiments, two aspects were mainly focused on, one is to extend the length of the filament, another one is to prolong the lifetime of laser-produced plasma. In the previous work[11], we have given out the nonequilibrium initial conditions of plasma channel evolution by calculating it's kinetic energy distribution after the laser pulse through combining the two aspects of separate researches in theory. Based on this calculation about the dynamics process formation mechanism of the plasma channel, in this paper, we pay attention on extending the length the plasma channel induced by the laser filament in practical experiment.

In order to realize the extension of the filament, researchers have adopted a series of laser modulation methods. The simplest way is to increase the energy of the femtosecond laser (fs-laser) pulse, while the filament extension is limited to a shorter length range [12]. For a certain pulse energy, there is a particular pulse duration corresponding to the longest filament[13]. And also, it is possible to obtain a longer filament by simultaneously controlling the spatial and temporal characteristics of 
the laser beam[14]. Recently, a new method by focusing ultrashort laser pulse with an axicon was used, the fs-laser can be changed into Bessel beam, then the longitudinal range of plasma channel can be generated[15][16]. The typical example is Maik Scheller et al., they used the axicon light road undertaking the role of the low-intensity "dressing" beam and acting as a distributed energy reservoir, this method continuously refuels the optical filament and extend light strings[17]. Also adding a conical and convergent circular Gauss beam around the filament can extend one order of the original filament was proposed in theoretically. H. C. et al, tuned the high-energy femtosecond pulse to match the perpendicular revivals of the molecular alignment, then the filament length was nearly doubled with respect to the randomly orientated molecules[18]. Another method is adjusting the spatial light modulator to generate multiple focus beams, and then the filament length can be doubled. However this method is not very practical since the calculation process is so complex by using spatial light modulator[19]. Integrating the advantages and disadvantages of these experiments, in this paper, we proposed a simple method that is a circular aperture quartz plate was used to realize the extension of the filament by modulating the center hole size and aperture thickness of the quartz plate. The influences of series parameters on the filamention were studied, and the moving-focus model was used to interpret the circular aperture quartz plate effect.

\section{Experiment}

The experiment set-up scheme used in this work is illustrated in Fig. 1. The laser system is equipped with a commercial fs-laser system (Ti: sapphire Micra 10 and regenerative amplifier Legend Elite-USP-HE, Coherent Corp. ) with $35 \mathrm{fs}$ pulse duration, $800 \mathrm{~nm}$ central wavelength, $3.5 \mathrm{~mJ} / \mathrm{pulse}$, and repetitive rate of $1 \mathrm{kHz}$, spot diameter of $8 \mathrm{~mm}$. The fs-laser firstly passes through a quartz plate with an outside diameter of $25 \mathrm{~mm}$ and a hole in the center. The inside diameter values of the central hole and the thickness of the quartz plate are changed in a series of variation values. After passing through the beam splitter, the reflected light is attenuated and enters the light spot analyzer to obtain the laser light intensity profile. The transmitted laser beam passes through a focusing lens with a focal length of $200 \mathrm{~mm}$. A focusing lens on the side of the filament is used to image the picture on the ICCD (PI-MAX3), thereby the length of the filament can be measured.

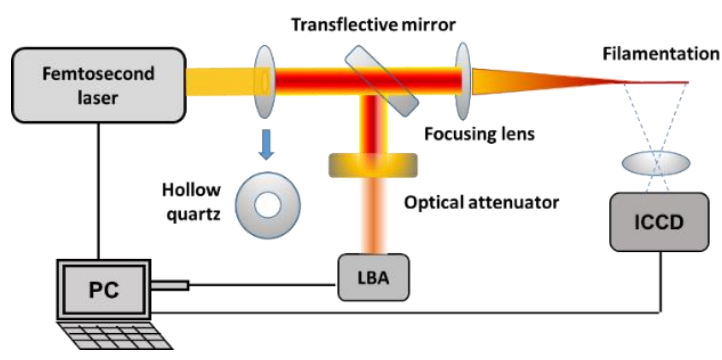

Figure 1. The laser filament extending experiment setup with circular aperture quartz plate

The experiment set-up scheme used in this work is illustrated in Fig. 1. The laser system is equipped with a commercial fs-laser system ( $\mathrm{Ti}$ : sapphire Micra 10 and regenerative amplifier Legend Elite-USP-HE, Coherent Corp. ) with $35 \mathrm{fs}$ pulse duration, $800 \mathrm{~nm}$ central wavelength, 3.5 $\mathrm{mJ} /$ pulse, and repetitive rate of $1 \mathrm{kHz}$, spot diameter of $8 \mathrm{~mm}$. The fs-laser firstly passes through a quartz plate with an outside diameter of $25 \mathrm{~mm}$ and a hole in the center. The inside diameter values of the central hole and the thickness of the quartz plate are changed in a series of variation values. After passing through the beam splitter, the reflected light is attenuated and enters the light spot analyzer to obtain the laser light intensity profile. The transmitted laser beam passes through a focusing lens with a focal length of $200 \mathrm{~mm}$. A focusing lens on the side of the filament is used to image the picture on the ICCD (PI-MAX3), thereby the length of the filament can be measured.

To compare the effects of the two type circular aperture quartz plates, the intensity distribution of the femtosecond laser pulse modulated by the circular aperture quartz plate was obtained by the spot analyzer (Spiricon, LBA-USB). 

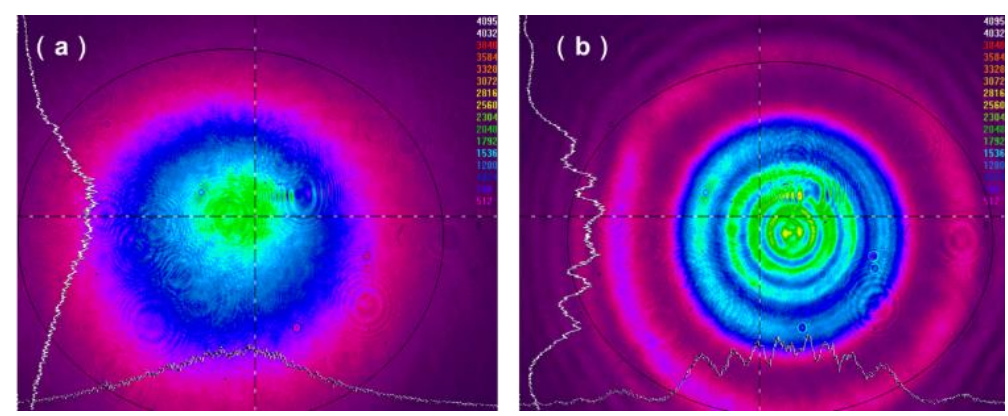

Figure 2. The intensity distribution of femtosecond laser spot after passing through the quartz plate (a) quartz plate without hole (b) the diameter of the hole is $4 \mathrm{~mm}$

Figure 2 gives out the comparison results, Fig. 2 (a) is the intensity distribution of femtosecond laser without modulation, the light spot envelope shows the Gaussian cross-section. Figure 2 (b) is the intensity distribution of the femtosecond laser passing through the modulated circular aperture quartz plate, the light spot envelope displays a ring-like distribution, and the projection in the horizontal and vertical direction is a fluctuating Gaussian like distribution.

\section{Results and disscussion}

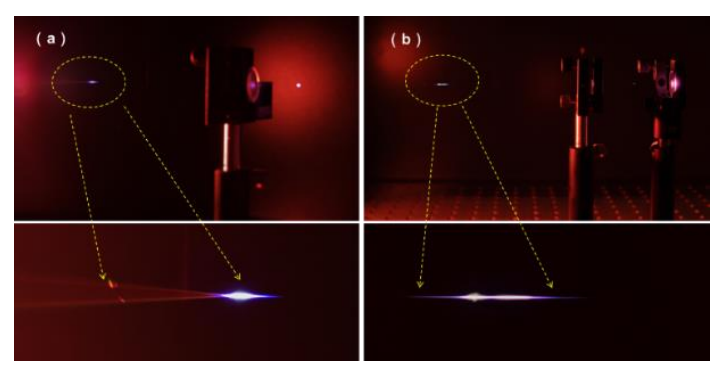

Figure 3. Images of fs-laser filament extension. (a) the normal quartz plate (b) the modulated circular aperture quartz plate

To give the intuitive visual effects, Fig. 3 gives fs-laser filamentation images of the above two situation captured by the digital camera, which show us a very beautiful brightness filamentation, and the length of the fs-laser filament was very obviously prolonged by using the modulated circular aperture quartz plate. For the further study, the accurate comparison was measured by the ICCD, the lens used here also were $200 \mathrm{~mm}$, the complete image of the fs-laser filament was obtained, as shown in fig. 4, which shows clearly that the length of fs-laser filament (fig.4 b, 320 pixel) by using the modulated circular aperture quartz plate is about 2.3 times as much as formed fs-laser filament without aperture (fig. 4 a, 140 pixel). From the picture, we also can see that the strongest position of the light filament moved forward about 80 pixel after using the circular aperture quartz plate, this is due to the energy redistribution in the laser filament.

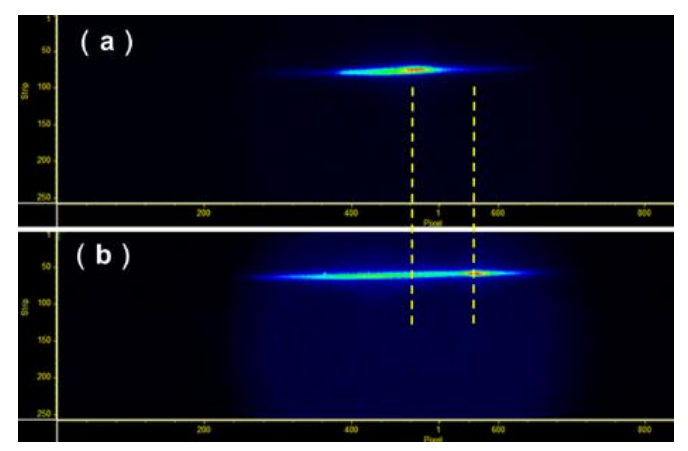


Figure 4. The image of filament obtained by ICCD, the focal length of the lens used are both $200 \mathrm{~mm}$

To discuss the detail effect of the modulated circular aperture quartz plate, two groups of comparison tests were done. We choose two different lens for comparison, the focal length were 17 $\mathrm{mm}$ and $20 \mathrm{~mm}$, and a series of optical fiament lengths were measured by the ICCD through changing the diameter of the hole in the circular aperture quartz plate, the experiment results are shown in Fig. 5.

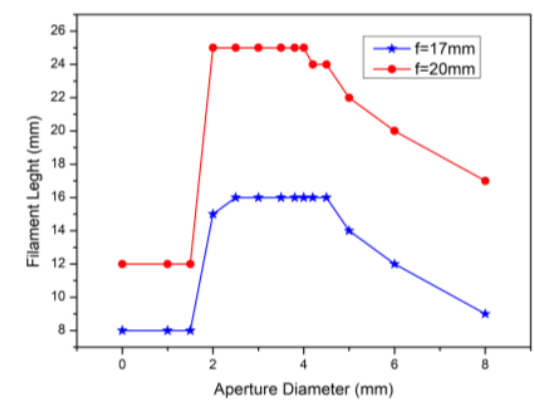

Figure 5. The length of the filament varies with the diameter of the aperture

From fig. 5, we can see that there is no obvious change in the length of the optical filament when the hole diameter change between $0 \mathrm{~mm}$ and $2 \mathrm{~mm}$. The filament is significantly prolonged when the aperture diameter is between $2 \mathrm{~mm}$ and $4.2 \mathrm{~mm}$. When the aperture diameter is $4.2 \mathrm{~mm}$ to $8 \mathrm{~mm}$, the length of the filament becomes shorter and shorter, return to the length without the aperture.
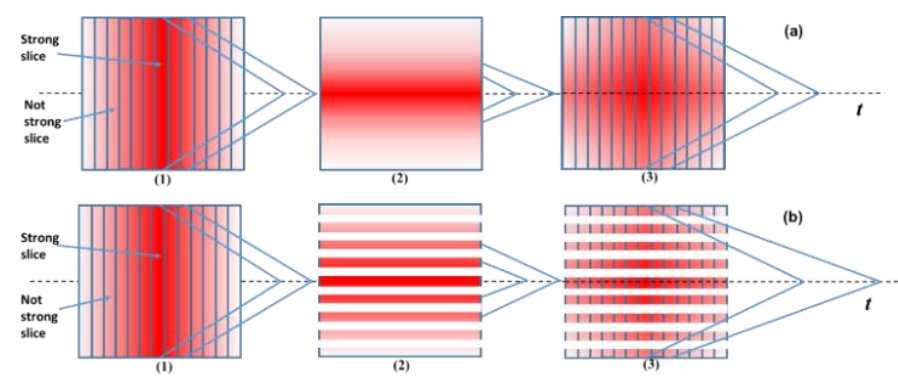

Figure 6. The schematic diagram of the moving-focus and self-focusing model

To better understand how the throughput of the optical filament be extended by the circular aperture quartz plate, the moving-focus and self-guided focusing model are used to explain it. Figure 6 shows the schematic diagram of the moving-focus and self-focusing model. The images (1) in fig. 6 (a) and 6 (b) are the moving-focus model, and the images (2) are the self-guided model, image (3) are the combination of the moving-focus and self-guided focusing model. After the circular aperture quartz plate modulation, the cross-section laser light redistribution, each ring is equivalent to a focal length of different focusing lens, different rings corresponding to different focus position, so the filament is extended.

The moving-focus model assumes that the laser pulses can be separated in time into many slices. Different slices with different energy are corresponding to different positions of the filament. In the time scale of femtosecond, the focal positions of different slices which do not exceed the critical selffocusing power $\mathrm{P}$ are almost at the same point, while the focusing positions of slices beyond the selffocusing threshold can be calculated according to the nonlinear wave equation. According to the nonlinear optics, we can get the following formula [20]

$$
z_{f}=\frac{0.367 k a^{2}}{\left\{\left[\left(P / P_{c r i t}\right)^{1 / 2}-0.852\right]^{2}-0.0219\right\}^{1 / 2}}
$$


Where $\mathrm{k}$ is the wave number, $\mathrm{a}$ is the beam radius at intensity of $1 / \mathrm{e}, \mathrm{P}$ is the incident laser power and $P_{c r i t}=3.77 \lambda^{2} / 8 \pi n_{0} n_{2}$ is the critical energy. According to this formula, different powers are corresponding to different focus positions, so that the laser is no longer focused on one point, but forms a sequence. In Fig. 6, the layer with highest power corresponds to the nearest position of the optical axis, the focal point of the adjacent layer corresponds to a position farther away from the nearest position, and so on. Simulation of the moving-focus model can successfully predict the generation and the end of the filament. According to the self-focusing effect, the stronger the light intensity, the greater the refractive index, and the closer the focusing position. Different laser intensities correspond to different focusing positions, which can be seen from Fig. 6. After passing through the quartz plate, the laser cross section becomes larger and the laser energy is redistributed. The inner ring energy is smaller than the original, so the position of the filament will be a bit farther, and the energy of outer ring is weaker, so the position of the corresponding filament is farther than the filament formed by the inner ring. Different rings act like lenses of different focal lengths, which produces a longer filament. In the meantime, we believe that the external ring will affect the energy reservoir of the filament form by the internal ring, this effect will make the overall extension of the left and right sides of the filament. Eventually, the effect shown in Fig. 4 appears. And also a plasma channel with $50 \mathrm{~cm}$ length was obtained as shown in Fig.7. It is noteworthy that when the hole diameter of the quartz plate is fixed, changing the thickness of the quartz or the depth of the hole, there is no significant change in the length of the filament.

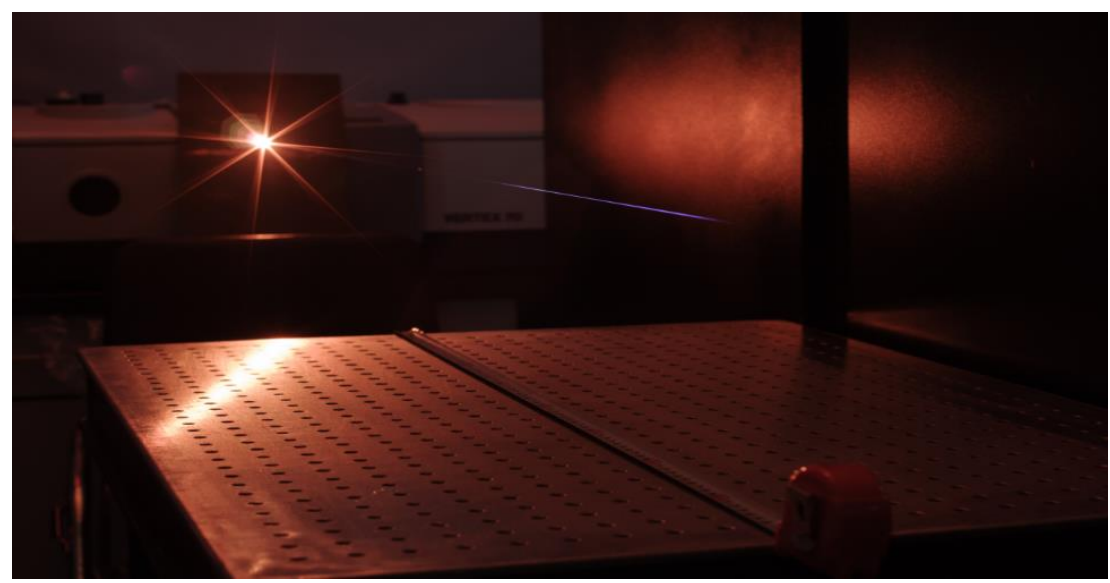

Figure 7. A plasma channel with $50 \mathrm{~cm}$ length obtained in experiment

\section{Conclusions}

In this paper, the effects of circular aperture quartz plate on the modulation of femtosecond laser spot and the length of femtosecond laser filament are studied. The ICCD and digital camera are used to measure image of femtosecond laser filament. The influence of hole diameter in different quartz plates on the length of filament was recorded. Under the laboratory conditions, the maximum diameter of the optical filament was obtained when the diameter of the hole in the quartz plate was $4 \mathrm{~mm}$. The extension of the light has nothing to do with the thickness of the quartz. The experimental results show that the circular aperture quartz plate can effectively prolong the optical filament produced by the femtosecond laser focusing. The circular aperture quartz plate has the advantages of simple fabrication, low cost and mass production, and is very practical in the femtosecond laser filament extension.

\section{Acknowledgements}

We gratefully acknowledge support for this work from the Beijing Commission of Education (Nos. KZ201710005004 and KM201810005001), and the National Key Research and Development Program (2018YFB0504400). 


\section{References}

1. A. Braun, G. Korn, X. Liu, D. Du, J. Squier, and G. Mourou 1995 Opt. Lett. 20, 73

2. P. Klarskov, A. C Strikwerda, K. Iwaszczuk, and P. U. Jepsen 2013 New J. Phys. 15, 075012

3. Z.Henis, G. Milikh, K. Papadopoulos, A. Zigler 2008 Journal of Applied Physics. 103(10), 47.

4. H. Cai, J. Wu, A. Couairon, H. Zeng 2009 Optics Letters. 34(6), 827

5. S. L. Chin, and K. Miyazaki 1999 Japanese journal of applied physics 38(4), 2011

6. J. Kasparian, R. Ackermann, Y. B. André, G. Méchain, G. Méjean, B. Prade, et al 2008 Optics Express. 16(8), 5757-5763

7. Q. Luo, H. L. Xu, S. A. Hosseini, J. F. Daigle, F. Théberge, M. Sharifi, et al 2006 Applied Physics B. 82(1), 105

8. D. S. Steingrube, E.Schulz, T. Binhammer, M. B. Gaarde, A. Couairon, U. Morgner, et al 2001 New Journal of Physics. 13(13), 043022

9. E. Goulielmakis, M. Schultze, M. Hofstetter, V. S. Yakovlev, J. Gagnon, M. Uiberacker, A. L. Aquila, E. M. Gul-likson, D. T. Attwood, R. Kienberger, F. Krausz, and U. Kleineberg 2008 Science. 320, 1614\

10. T. Popmintchev, H. C. Kapteyn,. Bright coherent ultrahigh harmonics in the kev x-ray regime from midinfrared femtosecond lasers. Science. 336(6086), 1287 (2012)

11. X. F. Shu, C. X. Yu, W. Li, S. B. Liu 2015 Physical Review A, 92(6) 063844

12. A. Talebpour, S. Petit, S. L. Chin 1999 Optics Communications. 171(4), 285

13. A. Couairon 2003 Applied Physics B: Lasers and Optics. 76(7), 789

14. Z. Jin, J.Zhang, M. H. Xu, X. Lu, Y. T. Li, Z. H. Wang, W. Yu 2005 Optics Express. 13(25), 10424

15. P. Polynkin, M. Kolesik, A. Roberts, D. Faccio, P. Di Trapani, J. Moloney 2008 Optics express. 16(20), 15733

16. S. Akturk, B. Zhou, M. Franco, A. Couairon, and A. Mysyrowicz 2009 Optics Communications. 282, 129

17. M. Scheller, M. S.Mills, M. A. Miri, W. Cheng, J. V. Moloney, M. Kolesik, D. N. Christodoulides 2014 Nature Photonics. 8(4), 297

18. M. S. Mills, M. Kolesik, D. N. Christodoulides 2013 Optics letters.38(1), 25

19. Z. Hong, Q. Zhang, S. A. Rezvani, P. Lan, P. Lu 2016 Optics Express. 24(4), 4029

20. A. Brodeur, C. Y. Chien, F. A. Ilkov, S. L. Chin, O. G. Kosareva, V. P. Kandidov 1997 Optics Letters. 22(5), 304 